\title{
Non-existence of wandering intervals and structure of topological attractors of one dimensional dynamical systems: 1 . The case of negative Schwarzian derivative
}

\author{
M. YU. LYUBICH \\ Steklov Mathematical Institute (Leningrad Department), Fontanka 27, \\ Leningrad 191011, USSR
}

(Received 10 November 1987 and revised 29 September 1988)

Abstract. It is proved that an arbitrary one dimensional dynamical system with negative Schwarzian derivative and non-degenerate critical points has no wandering intervals. This result implies a rather complete view of the dynamics of such a system. In particular, every minimal topological attractor is either a limit cycle, or a one dimensional manifold with boundary, or a solenoid. The orbit of a generic point tends to some minimal attractor.

\subsection{Statement of results}

Let $M$ be a one dimensional compact manifold with boundary, i.e. a finite union of disjoint intervals and circles (to consider non-connected $M$ is necessary for the proof, not for generality). Let us consider a class $\hat{O}_{d}$ of $C^{2}$-smooth maps $f: M \rightarrow M$ having $d$ critical points $c_{k} \in$ int $M$ (' $d$-modal') and satisfying the following conditions.

(U1) In punctured neighbourhoods of the critical points the following estimates hold

$$
A_{1}\left|x-c_{k}\right|^{\beta_{k}} \leq\left|f^{\prime}(x)\right| \leq A_{2}\left|x-c_{k}\right|^{\beta_{k}}
$$

where $A_{1}, A_{2}, \beta_{k}>0$.

(U2) Critical points $c_{k}$ are extrema.

(U3) The map $f$ is $C^{3}$-smooth and has negative Schwarzian derivative:

$$
S f=\frac{f^{\prime \prime}}{f^{\prime}}+\frac{3}{2}\left(\frac{f^{\prime \prime}}{f^{\prime}}\right)^{2}<0
$$

outside critical points.

Remark. If $x \in M$ belongs to a circle then $f^{(n)}(x)$ means the derivative with respect to the angular coordinate.

Let us also define the larger class $\mathscr{A}_{d}$ by only requiring that condition (U3) is satisfied locally, in some neighbourhood of the critical points. Set

$$
\mathscr{O}=\bigcup_{d=0}^{\infty} \mathcal{O}_{d}, \quad \mathscr{A}=\bigcup_{d=0}^{\infty} \mathscr{A}_{d} .
$$


Note that a $C^{\infty}$-smooth map $f$ belongs to $\mathscr{A}$ if all its critical points are non-flat extrema (i.e.

$$
\left.f^{\prime}\left(c_{k}\right)=f^{\prime \prime}\left(c_{k}\right)=\cdots=f^{\left(2 l_{k}-1\right)}\left(c_{k}\right)=0, \quad f^{\left(2 l_{k}\right)}\left(c_{k}\right) \neq 0 \quad \text { for some } l_{k}\right) .
$$

Denote by $f^{n}$ the $n$th iterate of $f$. A connected component $M_{0} \subset M$ will be called a rotation component if it is invariant under some iterate $f^{p}$ and $f^{p} \mid M_{0}$ is topologically conjugate to the irrational rotation of the circle. An interval $J$ is called wandering if $f^{n} J \cap f^{m} J=\varnothing$ for $n>m \geq 0$ and the orbit $\left\{f^{n} J\right\}_{n=0}^{\infty}$ does not tend to a cycle. An interval $J$ is called a homterval if $f^{n} \mid J$ is monotone for all $n \in \mathbb{N}=\{0,1,2, \ldots\}$. It is easy to see that every homterval is either wandering, or lies on a rotation component, or its orbit tends to a cycle. On the other hand, if $J$ is a wandering interval then $f^{n} J$ is a homterval for some $n \in \mathbb{N}$. The aim of the Part 1 of the present paper is to prove the following result.

MaIn theorem. (The case of negative Schwarzian derivative.) A map $f \in \mathcal{O}$ has no wandering intervals. In other words, any homterval which does not lie on a rotation component is attracted by a cycle.

The Main Theorem will be extended onto the smooth case in Part 2 which is due to A. M. Blokh and the author.

The Main Theorem solves an old problem. This topic goes back to the Poincarés paper dealing with homeomorphisms of the circle (see [18]). Since then efforts of a number of authors have been directed towards proving the non-existence of wandering intervals because their appearance complicates our understanding of the dynamics. Non-existence of wandering intervals was previously established in the following cases:

(1) for $C^{2}$-diffeomorphisms of the circle (Denjoy, see [18]);

(2) for unimodal $f \in \mathscr{O}_{1}$ (Guckenheimer [10]);

(3) for unimodal $f \in \mathscr{A}_{1}$ (de Melo and van Strien [15]);

(4) for $C^{\infty}$-smooth maps of the circle with non-flat critical points (Yoccoz [24]); this result is not a particular case of the Main Theorem since $f \in \mathscr{A}$ should satisfy (U2).

Remark that if we only require $C^{1}$-smoothness or allow flat critical points then wandering intervals may appear (see $[18,6,12])$. Some results on the behaviour of their orbits are obtained in $[3,4]$ (see Remark after Proposition 2 in the next section).

Similar problems arose in Faton-Julia's memoirs (1918-1920) on the conformal dynamics on the Riemann sphere. Non-existence of wandering domains in this case was proved in Sullivan's famous paper [21]. The method of quasi-conformal deformations used by Sullivan does not work in the one dimensional case. On the other hand, one of Fatou's results on the behaviour of orbits of hypothetic wandering domains ([7], pp. 60-61, see also [14]) became a starting point of our investigation.

Let $\omega(x)$ denote the limit set of the orbit $\left\{f^{n} x\right\}_{n=0}^{x}$. An invariant set $X \subset M$ will be called transitive if the map $f \mid X$ is topologically transitive, i.e. it has a dense orbit. Following Milnor [16], a closed invariant set $A \subset M$ will be called a topological attractor if 
(1) the realm of attraction $r l(A)=\{x: \omega(x) \subset A\}$ is a set of 2nd category (i.e. not 1st) in the sense of Baire;

(2) for any $A^{\prime} \varsubsetneqq A$ the set $\operatorname{rl}(A) \backslash r l\left(A^{\prime}\right)$ is also of the 2nd category.

A number of papers have been devoted to the investigation of attractors of one dimensional systems (Sharkovskii [19], Feigenbaum [8], Misiurewicz [17], Blokh $[1,2]$ etc,). The Main Theorem completes the description of the topological attractor's possible structure for the class of multi-modal maps under consideration. Namely there exist minimal attractors of only three kinds:

(1) $A$ is a limit cycle, i.e. the orbit of a periodic point such that int $r l(A) \neq \varnothing$;

(2) $A=\bigcup_{k=0}^{p-1} f^{k} I$ is an invariant transitive closed submanifold of $M$, i.e. $I$ is an interval or the circle,

$$
f^{k} I \cap f^{i} I=\varnothing \quad(0 \leq k<i \leq p-1), \quad f^{p} I \subset I
$$

and $f^{p} \mid I$ is topologically transitive;

(3) $A=\bigcap_{n=1}^{\infty} \bigcup_{k^{\prime \prime}=0}^{p^{-1}} f^{k} I_{n}$ is a solenoid. Here $I_{n}$ is a periodic interval of period $p_{n} \rightarrow \infty$, $I_{1} \supset I_{2} \supset \cdots$ and int $A \neq \varnothing$. In such a case $f: A \rightarrow A$ is topologically conjugate to the shift on a group.

Denote by $\operatorname{Per}(f)$ the set of periodic points of $f$. A point $x$ is called preperiodic if $f^{n} x \in \operatorname{Per}(f)$ for some $n$. By repeller we mean an invariant closed set $X \subset M$ such that int $X=\varnothing$ and $r l(X)=\bigcup_{n=0}^{\infty} f^{-n} X$.

Spectral decompositon of one dimensional dynamical systems (see [26] for the unimodal case and $[19,1,2]$ for the general continuous case) and the Main Theorem imply

Corollary 1.1. Let $f \in \mathcal{O}$. Then

$$
\overline{\operatorname{Per}(f)}=\bigcup A_{i} \cup R_{j},
$$

where $A_{i}$ are all attractors of kinds (1), (2), (3) and $R_{j}$ are some transitive repellers. For every $x \in M$ either $\omega(x) \subset A_{i}$ or $f^{n} x \in R_{j}$ for some $n \in \mathbb{N}$.

Corollary 1.2. For generic $x \in M$ (i.e. outside of a set of 1 st category) the limit set $\omega(x)$ is either a limit cycle, a transitive invariant submanifold or a solenoid.

Remark 1. By a rotation attractor we mean a cycle of rotation components of $M$. Let $l$ be the number of connected components of $M$ which are circles. Then a map $f \in \mathscr{O}_{d}$ may have at most $d+l+|\partial M|$ minimal attractors. Indeed, an attractor of kind (2) or (3) different from a rotation attractor must contain some critical point, while an attractor of kind (1) must attract some critical point or a boundary point (by Singer's theorem [20]).

Remark 2. The number of repellers $R_{j}$ in the decomposition (1) is in general infinite (at most countable). It is finite iff there are no solenoids. If $R_{j}$ does not contain a critical point then $f \mid R_{j}$ is topologically conjugate to the subshift of finite type.

Remark 3. Let $\Omega(f)$ denote the set of nonwandering points of $f$ (see [18]). Then it follows from $[1,2,6,25]$ that $\Omega(f)=\bigcup A_{i} \cup R_{j} \cup O_{k}$ where $A_{i}$ and $R_{j}$ are the components of the decomposition (1) and $O_{k}$ are the orbits of some preperiodic critical values. 
Remark 4. The intersection of any two components of the decomposition (1) is at most finite.

\section{Preliminaries}

Let $\lambda$ be the Lebesgue measure on $M$.

If $a, b$ lie in the same component $V$ of $M$ then $[a, b]$ denotes the (closed) interval ending at $a$ and $b$. Note that when $V$ is an interval, we do not assume $a \leq b$. If $V$ is a circle then it will always be clear which of two possible intervals ending at $a$, $b$ is considered. If $A, B$ are intervals then $[A, B]$ denotes the minimal closed interval containing $A$ and $B$ (with the same agreements as above). $[A, B)=\overline{[A, B] \backslash B}$ (note that $[A, B)$ is closed).

Denote by $C$ the set of critical points (extrema) of $f$ lying in int $M$. The points of $S=C \cup \partial M$ will be called singular.

In a small neighbourhood of any extremum $c$ define the involution $\tau: x \mapsto x^{\prime}$ as follows: $f\left(x^{\prime}\right)=f(x)$. By property (U1) of class $\sigma_{d}$ the involution $\tau$ is Lipschitz continuous. Denote by $L$ its Lipschitz constant.

In Part I we will assume that the following assumption holds.

AsSUMPTION A. There are no wandering intervals ending at singular points.

This technical assumption makes the main ideas of the proof much more transparent. Some remarks about the proof without Assumption A will be done in $\S 1.11$.

An interval $I$ will be called contractive if it is either wandering or all orbits originating in int $I$ tend to a limit cycle.

Proposition 1. If I is a non-contractive interval then

$$
\inf _{m \in \mathbb{N}} \lambda\left(f^{m} I\right)>0 .
$$

This proposition may be deduced from the view of the topological structure of one dimensional maps $[1,2]$ or proved directly by an easy argument.

The following statement is well-known.

Proposition 2. (See $[23,13])$. Let $f$ be a smooth one dimensional map having wandering interval $J$. Then $\omega(J)$ contains some critical point of $f$.

Remark. It is proved in $[3,4]$ that provided $f$ has negative Schwarzian derivative, there exists $c \in C$ such that $\omega(J)=\omega(c) \ni c$.

From now on we fix some maximal wandering homterval $J$ and some critical point $c \in \omega(J)$. Let $J_{n}=f^{n} J$. We say that $J_{m}$ is the $n$-nearest homterval to $c$ if $m \leq n$ and $J_{m}$ lies nearer to $c$ than all homtervals $J_{k}(k=0,1, \ldots, n, k \neq m)$. By 'nearer' we mean that $J_{k} \cap\left[J_{m}, \tau\left(J_{m}\right)\right]=\varnothing$. If $n=m$, we say simply 'the nearest homterval $J_{n}$ '. The idea of consideration the nearest homtervals in the unimodal case is due to Guckenheimer [10].

A homterval $I$ will be called solenoidal if for every $p$ there exists $n \in \mathbb{N}$ such that $J_{n}$ is contained in a periodic interval of minimal period more than $p$. We will prove the Main Theorem at first for non-solenoidal homtervals $(\$ 1.8)$ and then for solenoidal ones $(\$ 1.10)$. 
Now we define and establish to the end of the paper the large interger $\kappa \in \mathbb{N}$ and two small numbers $\eta>\boldsymbol{\xi}>0$.

Let $S_{p}$ be the set of periodic singular points, $S_{A}$ be the set of singular points attracting with some their neighbourhoods by limit cycles. Clearly $S_{p} \cap C \subset S_{A}$ and $c \notin S_{A}$.

Let $\kappa \in \mathbb{N}$ be so large that

(P1) $f^{m} a \neq b$ for any $a \in S, b \in S \backslash S_{p}, m \geq \kappa$.

Let $\eta>0$ be so small that

(P2) $\left|f^{\kappa} a-b\right|>\eta$ for any $a \in S, b \in S \backslash S_{p}$.

(P3) $\left|f^{m} a-c\right|>\eta$ for any $a \in S_{A} \cup S_{p}$ (it is possible since $c \notin S_{A}$ ).

Besides, we assume that $\eta$-neighbourhoods of singular points do not intersect and the involution $\tau$ is well-defined in $\eta$-neighbourhoods of critical points.

Finally, by Proposition 1 and Assumption A there exists $\xi \in(0, \eta)$ satisfying the following property.

(P4) Let $a \in S \backslash S_{A}, V$ be an interval containing $a$. Then

\subsection{Unimodal decomposition}

$$
\lambda(V) \geq \eta \Rightarrow \lambda\left(f^{m} V\right)>\xi \quad(m \in \mathbb{N}) .
$$

Let us consider a $(\delta \lambda(J))$-neighbourhood $U_{\delta}$ of the homterval $J$. Let $U_{\delta}^{ \pm}$be the components of $U_{\delta} \backslash J$. Since $J$ is the maximal homterval, we conclude by Proposition 1 that

$$
\lambda\left(f^{m} U_{\delta}^{ \pm}\right)>\rho(\delta)>0 \quad(m \in \mathbb{N})
$$

Now consider the sequence $J_{m_{i}} \rightarrow c$ of the nearest to $c$ homtervals. Set $\varepsilon=$ $\frac{1}{2} \min (\rho, \xi)$ where $\rho=\rho(\delta)$ and $\xi$ is defined in $\$ 1.2$. Choose from this sequence two homtervals $J_{s}$ and $J_{n}$ with large indices $s=m_{i}, n=m_{i+1}$ lying in the $\varepsilon$-neighbourhood of $c$ and so that

$$
\lambda\left(J_{n}\right)<\lambda\left(J_{s}\right) .
$$

Let $\alpha \in$ int $J_{s}$ lie farther from $c$ than the centre of $J_{s}$ and $\alpha \notin \bigcup_{n=1}^{\infty} f^{n} C$. Set $G=[\alpha, \tau(\alpha)]$. Then the inequalities $\lambda(G)<\rho$ and (1.1) imply $f^{n} U_{\delta} \not \subset G$. Let us consider the minimal neighbourhood $G_{0} \subset U_{\sigma}$ of $J$ such that $f^{n} a \notin$ int $G$ for $a \in \partial G_{0}$. Evidently

$$
\begin{gathered}
f^{n}\left(\text { int } G_{0}\right) \subset \text { int } G, \quad f^{n}\left(\partial G_{0}\right) \subset \partial G, \\
\lambda\left(Q_{0}^{ \pm}\right) / \lambda(J)<\delta,
\end{gathered}
$$

where $Q_{0}^{ \pm}$are the components of $G_{0} \backslash J$.

Further, consider those intervals of the orbit $\left\{f^{m} G_{0}\right\}_{m=0}^{n}$ which contain critical points: $f^{n_{i}} G_{0} \ni c_{i}\left(0<n_{1}<\cdots<n_{j} \leq n\right)$.

Let us show that

$$
\lambda\left(f^{n} G_{0}\right)<\eta
$$

If $n_{i}=n$, then we have $f^{n} G_{0} \subset G$ and $\lambda(G)<\eta$. Let $n_{i}<n$. Notice that $f^{n} G_{0}$ is mapped by $f^{n-n_{i}}$ into $G$ and $\lambda(G)<\xi$. By (P3) $c_{i} \notin S_{A}$. Hence (P4) implies (1.5).

It follows from (1.5) that we may consider the symmetrization $G_{i}=f^{n_{i}} G_{0} \cup$ $\tau\left(f^{n_{i}} G_{0}\right)$. If $n_{j}=n$ then set $k=j$ and note that $G_{k}=G$. If $n_{j}<n$ then set $k=j+1$, $n_{k}=n$ and $G_{k}=G$ by definition. 
In both cases we have constructed the sequence of closed intervals $G_{0}$, $G_{1}, \ldots, G_{k}=G$ and the sequence of integers $0=n_{0}<n_{1}<\cdots<n_{k}=n$ such that

(D1) $G_{i}$ lie in $\eta$-neighbourhood of $c_{i}$ for $i=1,2, \ldots, k$ and $\tau\left(G_{i}\right)=G_{i}$;

(D2) $J_{n_{i}} \subset$ int $G_{i}, G_{k}$ does not contain homtervals $J_{l}$ for $l<n$;

(D3) $f^{\prime} G_{i} \subset G_{i+1}, f^{\prime}\left(\partial G_{i}\right) \subset \partial G_{i+1}$ for $l_{i}=n_{i+1}-n_{i}$;

(D4) $f^{i} \mid G_{0}$ is monotone and $f^{\prime} \mid G_{i}$ are unimodal for $i \geq 1$.

In such a case we will say that the unimodal decomposition of $f^{n} \mid G_{0}$ determined by the sequence $\left\{G_{i}, n_{i}\right\}_{i=0}^{k}$ is given. We call $k$ the order of the decomposition and $f^{n_{i+1}-n_{i}}$ the factors. The maximal order of decompositions $\left\{G_{i}, n_{i}\right\}_{i=0}^{k}$ for which $n_{k}=n$ will be denoted by ord $\left(J_{n}\right) \equiv$ ord $(n)$.

Remark. Instead of (D1) one may require

$\left(\mathrm{D}^{0} 1\right) G_{i} \ni c_{i}, \tau\left(G_{i}\right)=G_{i}$ and $\lambda\left(G_{k}\right)<\xi$.

In fact, the implication $\left(D^{0} 1\right) \Rightarrow(D 1)$ was proved above (see the proof of inequality (1.5)).

Finally, let us complete the constructed decomposition of $f^{n} \mid G_{0}$ to the maximal one. For a new decomposition we retain the notations $\left\{G_{i}, n_{i}\right\}_{i=0}^{k}$.

\subsection{Expanding property}

In the last section we constructed the maximal unimodal decomposition $\left\{G_{i}, n_{i}\right\}_{i=0}^{k}$. Set $T_{0}=G_{0}, G_{i}=\left[z_{i}, \tau\left(z_{i}\right)\right]$ for $i \geq 1$. For $i \geq 1$ satisfying $n_{i+1}-n_{i}>\kappa$ put $\nu_{i}=$ $n_{i+1}-n_{i}-\kappa$. Consider the maximal $T_{i}=\left[f^{\kappa} z_{i}, \zeta_{i}\right]$ ending at $f^{\kappa} z_{i}$ and containing $f^{\kappa} G_{i}$ on which $f^{\nu_{i}}$ is monotone. Let $R_{i} \equiv\left(J_{n_{i}+\kappa}, \zeta_{i}\right]$ (see figure 1 ).

The following lemma may be considered as the multi-modal version of Lemma 3 of [5] which is due to A. M. Blokh.

LEMMA 1.1. Let $\left\{G_{i}, n_{i}\right\}_{i=0}^{k}$ be the maximal unimodal decomposition and $\lambda\left(G_{k}\right)<\xi$. If $n_{i+1}-n_{i}>\kappa$ then

$$
f^{\nu} T_{i} \supset G_{i+1} .
$$

Proof. For $i=0$ the map $f^{\nu_{0}}\left|T_{0}=f^{n_{\mathrm{i}}-n_{01}}\right| G_{0}$ is monotone and $f^{\nu_{0}}\left(\partial T_{0}\right) \subset \partial G_{1}$. Hence $f^{\nu_{0}} T_{0}=G_{1}$.

Further let $i \geq 1$. Assume that the statement of the Lemma fails. Then $f^{\nu} T_{i} \subset G_{i+1}$. The endpoint $\zeta_{i}$ of $T_{i}$ is either a preimage of some critical point or a boundary point.

In fact, the latter case is excluded. Indeed, otherwise it follows from (P3) (see $\S 1.2)$ that $\zeta_{i} \notin S_{A} \cup S_{P}$. Therefore by (P4):

$$
\lambda\left(R_{i}\right)>\eta \Rightarrow \lambda\left(f^{n-n_{i}-\kappa} R_{i}\right)>\xi
$$

The inequality in the left-hand side is true by (P2) since $\lambda\left(R_{i}\right) \geq\left|f^{\kappa} c_{i}-\zeta_{i}\right|$. The inequality in the right-hand side fails since $f^{n-n_{i}-\kappa} R_{i} \subset G_{k}$. So we obtain a contradiction.

Thus, $\zeta_{i}$ is a preimage of some critical point $a$. So $a$ is an endpoint of an interval $V=f^{t} T_{i}$ for some $t \in\left(0, \nu_{i}\right)$. Then $f^{n-r} V \subset G_{k}$ where $r=n_{i}+\kappa+t$. Applying (P3) and (P4) to $f^{n-r}$ we obtain $\lambda(V)<\eta$. Hence, we may consider the symmetric interval $H=V \cup \tau(V)$ containing $J_{r}$ (see figure 1). It is easy to see that the sequences $\left\{G_{0}, \ldots, G_{i}, H, G_{i+1}, \ldots, G_{k}\right\}$ and $\left\{n_{0}, \ldots, n_{i}, r, n_{i+1}, \ldots, n\right\}$ give a new unimodal 

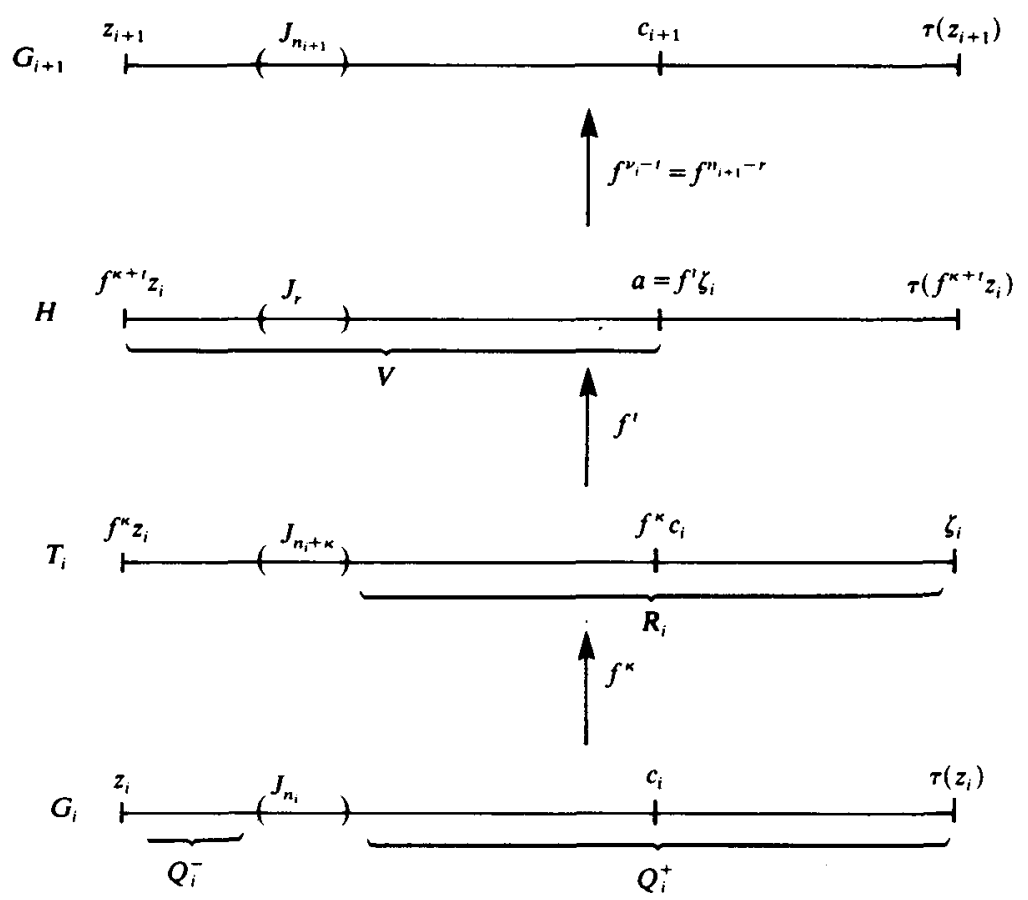

FIGURE 1

decomposition of $f^{n} \mid G_{0}$. This contradicts the maximality of the initial decomposition.

\subsection{Distortion lemmas}

First let us state the elementary.

THE FIRST DISTORTION LEMMA. Let $f$ be a $C^{1}$-smooth map satisfying the Property $(U 1)$. Let $U, W$ be the intervals having the common endpoint which do not contain critical points, int $U \cap$ int $W=\varnothing$. If $\lambda(U) \leq \lambda(W)$ then

$$
\frac{\lambda(f U)}{\lambda(f W)}: \frac{\lambda(U)}{\lambda(W)} \leq A(f) .
$$

Proof. Fix a small $\varepsilon>0$. If $\lambda(W) \geq \varepsilon$ then $\lambda(f W) / \lambda(W) \geq D>0$. Besides $\lambda(f U) / \lambda(U) \leq\left\|f^{\prime}\right\|$, and we obtain (1.7).

Let $\lambda(W)<\varepsilon, X$ be an $\varepsilon$-neighbourhood of $C$. We have

$$
\frac{\lambda(f U)}{\lambda(U)}: \frac{\lambda(f W)}{\lambda(W)}=\frac{\left|f^{\prime}(x)\right|}{\left|f^{\prime}(y)\right|}, \quad x \in U, y \in W .
$$

If $W \cap X=\varnothing$ then (1.8) implies (1.7).

Let $W \cap X \neq \varnothing$. Then $U \cup W$ lies in the $3 \varepsilon$-neighbourhood of some critical point c. Divide $W$ into two half-intervals $W_{1}$ and $W_{2}$ where $W_{1}$ has a common point with 
U. We have

$$
\frac{\lambda(f U)}{\lambda(U)}: \frac{\lambda(f W)}{\lambda(W)} \leq 2 \frac{\lambda(f U)}{\lambda(U)}: \frac{\lambda\left(f W_{1}\right)}{\lambda\left(W_{1}\right)}=2 \frac{\left|f^{\prime}(x)\right|}{\left|f^{\prime}(y)\right|},
$$

where $x \in U, y \in W_{1}$. But by (U1) $\left|f^{\prime}(z)\right| \asymp|z-c|^{\beta}$ holds in the $3 \varepsilon$-neighbourhood of $c$. This immediately implies (1.7) in the case when $W$ lies farther from $c$ than $U$. Let $W$ lie nearer to $c$ than $U$. Then setting $\rho=\operatorname{dist}(c, W)$ we obtain

$$
\begin{aligned}
\frac{\left|f^{\prime}(x)\right|}{\left|f^{\prime}(y)\right|} \asymp \frac{|x-c|^{\beta}}{|y-c|^{\beta}} & \leq\left(\frac{\lambda(U)+\lambda(W)+\rho}{\lambda\left(W_{2}\right)+\rho}\right)^{\beta} \\
& \leq\left(\frac{4 \lambda\left(W_{2}\right)+\rho}{\lambda\left(W_{2}\right)+\rho}\right)^{\beta} \leq 4^{\beta} .
\end{aligned}
$$

The Lemma is proved.

This Lemma is the only place in Part 1 of the paper where we use the full power of the estimates (U1) rather than just the Lipschitz of $\tau$.

Further in this section $\varphi:[0,1] \rightarrow[0,1]$ will be the $C^{3}$-smooth surjective map satisfying

(1) $\varphi$ has no critical points in $[0,1]$;

(2) $\varphi$ has negative Schwarzian derivative.

The following result is similar to the Köebe Distortion Theorem for univalent functions (see [9]).

The Köebe Property $[22,11]$. Let $\delta>0$. If $\varphi(x), \varphi(y) \in[\delta, 1-\delta]$ then $\left|\varphi^{\prime}(x)\right| /\left|\varphi^{\prime}(y)\right| \leq B(\delta)$ where $B(\delta)$ does not depend on $\varphi$.

Divide $[0,1]$ into the union of three intervals $\mathrm{H}^{-} \cup K \cup \mathrm{H}^{+}$(where $K$ lies between $H^{-}$and $\mathrm{H}^{+}$). The Köebe Property immediately implies

THE SECOND DISTORTION LEMMA. There exists a function $\gamma: \mathbb{R}_{+} \rightarrow \mathbb{R}_{+}$such that

$$
\text { if } \frac{\lambda\left(\varphi H^{+}\right)}{\lambda(\varphi K)} \geq \alpha \text { and } \frac{\lambda\left(\varphi H^{-}\right)}{\lambda(\varphi K)} \geq \alpha \text { then } \frac{\lambda\left(H^{ \pm}\right)}{\lambda(K)} \geq \gamma(\alpha) \text {. }
$$

This Distortion Lemma is the main analytical tool in Part 1 of the present paper.

\subsection{Distortion estimates for unimodal factors}

Let $\left\{G_{i}, n_{i}\right\}_{i=0}^{k}$ be the unimodal decomposition of $f^{n}$. Denote by $Q_{i}^{ \pm}$the connected components of $G_{i} \backslash J_{n_{i}}$. Moreover, for $i \geq 1$ let $Q_{i}^{-}$be that component which does not contain $c_{i}$.

LemmA 1.2. Let $f \in \mathcal{O}$. There exists a function $\sigma(\alpha)>0$ such that

$$
\lambda\left(Q_{i+1}^{-}\right) / \lambda\left(J_{n_{i+1}}\right) \geq \alpha \Rightarrow \lambda\left(Q_{i}^{ \pm}\right) / \lambda\left(J_{n_{i}}\right) \geq \sigma(\alpha) .
$$

Remark 1. Lemma 1.2 is the unique step of the proof for which the condition of negative Schwarzian derivative is essential. The main problem of Part 2 will be to obtain its smooth analogue. 
Remark 2. The function $\sigma(\alpha)$ depends on $f$ but is independent of decomposition $\left\{G_{i}, n_{i}\right\}_{i=0}^{k}$. One may think also that $\sigma(\alpha) \leqslant \alpha$ and $\sigma(\alpha)$ is monotone decreasing.

Proof. Assume first that $i \geq 1$ and $n_{i+1}-n_{i}>\kappa$. Since $Q_{i}^{+} \supset \tau\left(J_{n_{i}}\right)$ for $i \geq 1$, $\lambda\left(Q_{i}^{+}\right) / \lambda\left(J_{n_{i}}\right) \geq L^{-1}$. So, only the estimate for $\lambda\left(Q_{i}^{-}\right) / \lambda\left(J_{n_{i}}\right)$ is non-trivial. To prove it let us consider the map $f^{\nu_{i}} \mid T_{i}$ (see $\S 4$ and figure 1 ).

By Lemma $1.1 f^{\nu_{i}} T_{i} \supset G_{i+1}$ and we may apply the Second Distortion Lemma to the partition $T_{i}=f^{\kappa} Q_{i}^{-} \cup J_{n_{i}+\kappa} \cup R_{i}$. It gives

$$
\lambda\left(f^{\kappa} Q_{i}^{-}\right) / \lambda\left(J_{n_{i}+\kappa}\right) \geq \gamma\left(\min \left(\alpha, L^{-1}\right)\right)=\tilde{\gamma}(\alpha) .
$$

Applying now the First Distortion Lemma to $f^{\star}$, we obtain

$$
\lambda\left(Q_{i}^{-}\right) / \lambda\left(J_{n_{i}}\right) \geq \min \left(1, A^{-1} \tilde{\gamma}(\alpha)\right)
$$

for $A=A\left(f^{\kappa}\right)$.

So, (1.9) is proved under the assumptions $i \geq 1$ and $n_{i+1}-n_{i}>\kappa$. Without these assumptions the proof is still more simple. Namely, in the case $i=0(1.9)$ follows directly from the Second Distortion Lemma. In the case $n_{i+1}-n_{i} \leq \kappa$ it follows from the First Distortion Lemma.

\subsection{Decompositions of low order}

In this section we assume that $J$ is non-solenoidal homterval (see $\S 1.2$ ). Equivalently, if $a \in \omega(J)$ then $a$ has no small periodic neighbourhoods. Then one may a priori choose $\eta$ so small that the following property holds (in addition to $\left(\mathrm{P}_{2}\right),\left(\mathrm{P}_{3}\right)$ ):

(P5) If $J_{m}$ is contained in an $\eta$-neighbourhood $U_{\eta}(a)$ of an extremum $a$ for some $m \in \mathbb{N}$, then $U_{\eta}(a)$ is a non-periodic interval.

The following Lemma shows that in the non-solenoidal case the length of any unimodal decomposition does not exceed $d$.

LEMMA 1.3. Let $J$ be a non-solenoidal wandering homterval. Consider the unimodal decomposition $\left\{G_{i}, n_{i}\right\}_{i=0}^{k}, c_{i} \in G_{i}$ for $i \geq 1$. Then

(a) $J_{n_{i}}$ are the $n$-nearest homtervals to $c_{i}(i \geq 1)$;

(b) critical points $c_{i}$ are pairwise distinct;

(c) $k \leq d$.

Proof. The implications (a) $\Rightarrow(\mathrm{b}) \Rightarrow$ (c) are trivial. Let us prove (a). Assume that $J_{\boldsymbol{I}}$ lies nearer to $c_{i}$ than $J_{n_{i}}$ for some $i \in[1, k], l \in[0, n]$. Then $i<k, l<n$ by property (D2) $(\$ 1.3)$. Now consider two cases.

(i) $l<n_{i}$. Then

$$
G_{k} \supset f^{n-n_{i}} G_{i} \supset f^{n-n_{i}} J_{l}=J_{n-\left(n_{i}-l\right)}
$$

which contradicts (D2).

(ii) $l>n_{i}$. Put $p=l-n_{i} \in\left(0, n-n_{i}\right)$ and consider $f^{p} \mid G_{i}$. By (P5) $f^{p} G_{i} \not \subset G_{i}$. Then $f^{p} G_{i}$ contains $J_{n_{i}}$ or $\tau\left(J_{n_{i}}\right)$ and hence $f^{p+1} G_{i} \supset J_{n_{i}+1}$. Applying $f^{n-l-1}$ to the last inclusion we obtain

$$
J_{n-p}=f^{n-1-1} J_{n_{i}+1} \subset f^{n-1-1}\left(f^{p+1} G_{i}\right)=f^{n-n_{i}} G_{i} .
$$

Hence $J_{n-p} \subset G_{k}$ which contradicts property (D2) of the decomposition. 


\subsection{Absence of non-solenoidal homtervals for $f \in \mathcal{O}$}

Recall that $Q_{i}^{ \pm}$denotes the connected components of $G_{i} \backslash J_{n_{i}}$ (see $\S 1.6$ ). By the definition of $G_{k} \equiv G$ (see $\S 1.3$ ), inequality (1.2) and Lipschitz continuity of $\tau$, we obtain

$$
\frac{\lambda\left(Q_{k}^{-}\right)}{\lambda\left(J_{n_{k}}\right)} \geq \frac{\lambda\left(J_{s}\right) / 2}{\lambda\left(J_{n}\right)} \geq \frac{1}{2}, \quad \frac{\lambda\left(Q_{k}^{+}\right)}{\lambda\left(J_{n_{k}}\right)} \geq \frac{\lambda\left(\tau\left(J_{n}\right)\right)}{\lambda\left(J_{n}\right)} \geq \frac{1}{L} .
$$

Applying Lemma $6.1 k$ times moving along the chain $\left\{G_{i}\right\}_{i=0}^{k}$, we conclude

$$
\lambda\left(Q_{0}^{ \pm}\right) / \lambda(J) \geq \sigma^{0 k}(1 / 2 L)
$$

But by Lemma $1.3 k \leq d$. Now return to the inequality (1.4) in $\S 1.3$. One may a priori choose $\delta$ so that $\delta<\sigma^{\circ d}(1 / 2 L)$. Then the inequalities (1.4) and (1.10) are contradictory.

\subsection{Decompositions of high order}

The absence of solenoidal homtervals we prove by induction. The trivial case $d=0$ gives the base of induction. Assume that $d>0$ and maps $f \in \mathscr{O}_{k}$ have no wandering homtervals for $k \leq d-1$. Then the structure of unimodal decompositions $\left\{G_{i}, n_{i}\right\}_{i=0}^{k}$ of high order must be very special. We describe it in the following two Lemmas.

LEMMA 1.4. (a) The sequence of critical points $c_{i} \in G_{i}$ is periodic of minimal period $d$;

(b) $J_{n_{i}}$ is the $\left(n_{i+d}-1\right)$ nearest to $c_{i}$ homterval for $i \leq k-d$.

Let $H_{n}$ be the maximal interval containing $J$ on which $f^{n}$ is monotone, $M_{n}=f^{n} H_{n}$. Denote by $M_{n_{j}}^{-}$the component of $M_{n} \backslash J_{n_{i}}$ lying farther from $c_{i}$ than $J_{n_{i}}$ and by $M_{n_{i}}^{+}$ the other component. Set $F_{i}=\left[J_{n_{i}}, \tau\left(J_{n_{i}}\right)\right]$.

LEMMA 1.5. There exists $\gamma$ (independent of the decomposition) such that provided $n_{i} \geq \gamma$ and $d \leq i \leq k-d$ we have

(a) $M_{n_{i}}$ contains $J_{n_{i-d}}$ or $\tau\left(J_{n_{i-d}}\right)$;

(b) $J_{n_{i}} \cup M_{n_{i}}^{+} \supset F_{i} \cap f^{n_{i}-n_{i-1}} F_{i-1} \ni c_{i}$.

Proof of Lemma 1.4. Let $\left\{G_{i}, n_{i}\right\}_{i=0}^{k}$ be the unimodal decomposition. First observe that $G_{i}$ does not contain homtervals $J_{l}$ for $l<n_{i}$. Indeed, otherwise $G_{k} \supset J_{n_{k}-\left(n_{i}-l\right)}$ which contradicts the property (D2) (cf case (i) of the proof of Lemma 1.3). Hence $J_{n_{i}}$ is the nearest to $c_{i}$ homterval.

Further, let $c_{i}, c_{i+1}, \ldots, c_{l-1}$ be pairwise distinct critical points, while $c_{i}=c_{i}$. Then $J_{n_{1}}$ lies nearer to $c_{l}$ than $J_{n_{i}}$. We state that

$$
f^{n_{1}-n_{i}} G_{i} \subset \text { int } F_{i} \text {. }
$$

Indeed, otherwise one may check that $G_{k} \supset J_{n_{k}-\left(n_{i}-n_{i}\right)}$ which contradicts (D2) again (cf case (ii) of the proof of Lemma 1.3).

It follows from (1.11) that $F_{i}$ is a periodic interval of period $n_{i}-n_{i}$. Hence, the union of intervals

$$
O_{i, 1}=\bigcup_{m=0}^{n_{1}-n_{i}-1} f^{m} F_{i}
$$


is $f$-invariant. By induction, $f \mid O_{i, l}$ should have at least $d$ critical points. Hence $l-i=d$ and $\left\{c_{i}, \ldots, c_{l-1}\right\}=C$. Now Lemma 1.4(a) follows.

To prove Lemma 1.4(b) assume that $J_{l}$ lies nearer to $c_{i}$ for some $l<n_{i+d}$. This leads to a contradiction by the same argument as used just now. We omit the details.

Proof of Lemma 1.5. The proof of statements (a) and (b) are based upon similar ideas and we restrict ourselves to the proof of (b).

Let us show that

$$
\text { int }\left(f^{n_{i+1}-n_{i}} F_{i}\right) \ni c_{i+1} \quad(1 \leq i \leq k-d, 1 \leq l \leq d) .
$$

Indeed, if (1.12) fails for some $l<d$ then $f \mid O_{i, i+d}$ has less than $d$ critical points. If (1.12) fails for $l=d$ then $f^{n_{i+d}-n_{i}} F_{i}^{\nu} \subset F_{i}^{\nu}$ for some component $F_{i}^{\nu}$ of $F_{i} \backslash\left\{c_{i}\right\}$. Hence $f \mid O_{i, i+d}^{\nu}$ has less than $d$ critical points where

$$
O_{i, 1}^{\nu}=\bigcup_{m=0}^{n_{i}-n_{i j}-1} f^{m} F_{i}^{\nu}
$$

In both cases we arrive at a contradiction which proves (1.12).

Let $\gamma$ be defined by the property that $\partial H_{\gamma} \not \subset \partial M$. Then the endpoints of $H_{n_{i}}$ are preimages of critical points for $n_{i} \geq \gamma$. So there exists $t \in\left(0, n_{i}\right)$ such that $f^{t} H_{n_{i}} \equiv V$ ends at some critical point $c_{j}$ for $i-d \leq j<i$. Now consider two cases:

(i) $t>n_{j}$. Since $t<n_{i} \leq n_{j+d}$, by Lemma $1.4 V \cup \tau(V)=\left[J_{t}, \tau\left(J_{t}\right)\right] \supset J_{n_{i}}$. Applying $f^{n_{i}-t}$ we conclude $M_{n_{i}}^{+} \supset J_{n_{i}-\left(t-n_{i}\right)}$. But $F_{i}$ does not contain any interval $J_{l}$ for $l<n_{i}$. Hence $M_{n_{i}}^{+} \supset F_{i}$.

(ii) $t \leq n_{j}$. Since $f^{n_{i}-t} \mid V$ is monotone, $f^{n_{i}-n_{i}} \mid F_{j}$ is unimodal. It follows from (1.12) that $j=i-1$ and $t=n_{i-1}$. Hence $M_{n_{i}}^{+}=f^{n_{i}-n_{i-1}} F_{i-1}$ and we are done.

\subsection{Absence of solenoidal homtervals for $f \in \mathcal{O}$}

We may a priori choose $\eta$ as follows:

(P6) If $J_{m}$ is $\eta$-close to a critical point $a \in C$ for some $m \in \mathbb{N}$ then $a \in \omega(J)$.

The proof of the Main Theorem in the non-solenoidal case shows that there exist unimodal decompositions $\left\{G_{i}, n_{i}\right\}_{i=0}^{k}$ of arbitrarily large order $k$. It follows from Lemma 1.4 and (P6) that in such a case all critical points $c_{j} \in C$ belong to $\omega(J)$. Let $N_{j} \subset \mathbb{N}$ be the sequence of numbers $m$ for which $J_{m}$ are the nearest to $c_{j}$ homtervals. We have $J_{m} \rightarrow c_{j}\left(m \rightarrow \infty, m \in N_{j}\right)$. Now let us consider two cases:

(i) For some $j$ the sequence $\left\{\lambda\left(J_{m}\right)\right\}_{m \in N_{j}}$ is not asymptotically monotone. Let $N_{j}=$ $\left\{m_{i}\right\}_{i=1}^{\infty}$. Then there exists arbitrarily large $s$ satisfying

$$
\lambda\left(J_{m_{x}}\right) \geq \lambda\left(J_{m_{x+1}}\right) .
$$

Let $a_{s} \in$ int $J_{m_{s}}$ lie farther from $c_{j}$ then the centre of $J_{m_{s}}$, and $a_{s} \notin \bigcup_{n=1}^{\infty} f^{n} C$. Consider the interval $G=\left[a_{s}, \tau\left(a_{s}\right)\right]$ and construct a maximal unimodal decomposition $\left\{G_{i}, n_{i}\right\}_{i=0}^{k}$ for which $G_{k}=G, n_{k}=m_{s+1}$ (see \& 1.3). Lemma 1.2 implies

$$
\lambda\left(Q_{0}^{ \pm}\right) / \lambda(J) \geq \sigma^{\circ k}\left[(2 L)^{-1} \lambda\left(J_{m_{s}}\right) / \lambda\left(J_{m_{x+1}}\right)\right] \geq \sigma^{\circ k}(1 / 2 L) .
$$

As $\lambda\left(Q_{0}^{ \pm}\right) / \lambda(J) \rightarrow 0(s \rightarrow \infty)$, we obtain $k=$ ord $\left(m_{s+1}\right) \rightarrow \infty(s \rightarrow \infty)$ (we mean now and further that $s$ satisfies (1.13)). 
By Lemma $1.4 n_{k-j d}=m_{s+1-j}(j=1,2, \ldots)$. Applying Lemma 1.2 to the chain $\left\{G_{i}, n_{i}\right\}_{i=k-2 d}^{k}$, we obtain

$$
\lambda\left(Q_{k-2 d}^{-}\right) / \lambda\left(J_{m_{v-1}}\right) \geq \sigma^{\circ 2 d}(1 / 2 L) \equiv \rho .
$$

This inequality and Lemma $1.5(\mathrm{a})$ imply

$$
\lambda\left(M_{m_{s-1}}^{-}\right) / \lambda\left(J_{m_{s-1}}\right) \geq \rho .
$$

On the other hand, Lemma $1.5(\mathrm{~b})$ implies

$$
\begin{aligned}
\lambda\left(M_{m_{s-1}}^{+}\right) / \lambda\left(J_{m_{s-1}}\right) & \geq \lambda\left(J_{m_{s-1}}, c\right] / \lambda\left(J_{m_{x-1}}\right) \\
& \geq L^{-1} \lambda\left(J_{m_{x}}\right) / \lambda\left(J_{m_{s-1}}\right) \geq L^{-1} .
\end{aligned}
$$

The estimates (1.14) amd (1.15) are uniform with respect to $s$. This contradicts the Second Distortion Lemma applied to $f^{m_{s-1}} \mid H_{m_{x-1}}$, since $\lambda\left(H_{m_{s-1}}^{ \pm}\right) / \lambda(J) \rightarrow 0(s \rightarrow \infty)$.

(ii) The sequences $\left\{\lambda\left(J_{m}\right)\right\}_{m \in N_{i}}$ are monotone for sufficiently large $m$. By the same argument as in (i) we obtain ord $(m) \rightarrow \infty(m \rightarrow \infty)$. Then using Lemmas 1.4 and 1.5 we see

(a) there exists the unified numeration of the sequence $\left\{J_{m}\right\}_{m \in \cup N_{j}}=\left\{J_{n_{i}}\right\}_{i=1}^{\infty}$ such that $\left\{n_{j+i d}\right\}_{i=0}^{\infty}=N_{j}$ and the sequence $\left\{n_{i}\right\}_{i=1}^{\infty}$ is monotonically increasing;

(b) $M_{n_{i}} \supset\left[J_{n_{i-d}}, c_{j}\right]$ for $i \equiv j(\bmod d)$.

Now consider two sub-cases

(ii) there exist arbitrarily large $n_{i}$ for which $J_{n_{i}} \cup M_{n_{i}}^{+} \supset F_{i}$. Then

$$
\lambda\left(M_{n_{i}}^{+}\right) / \lambda\left(J_{n_{i}}\right) \geq \lambda\left(\tau\left(J_{n_{i}}\right)\right) / \lambda\left(J_{n_{i}}\right) \geq L^{-1} .
$$

Besides, by property (b)

$$
\lambda\left(M_{n_{i}}^{-}\right) / \lambda\left(J_{n_{i}}\right) \geq L^{-1} \lambda\left(J_{n_{i-d}}\right) / \lambda\left(J_{n_{i}}\right) \geq L^{-1} .
$$

For large $i$ the last two inequalities contradict the Second Distortion Lemma applied to $f^{n_{i}} \mid H_{n_{i}}$.

(ii $\left.{ }_{2}\right) J_{n_{i}} \cup M_{n_{i}}^{+} \subset F_{i}$ for all sufficiently large $i$. Then by Lemma 1.5

$$
f^{n_{i+1}-n_{i}} F_{i} \subset J_{n_{i+1}} \cup M_{n_{i+1}}^{+} \subset F_{i+1} .
$$

Hence

$$
f^{n_{i+d}-n_{i}} F_{i} \subset F_{i+d}
$$

In particular, $f^{n_{i+d}-n_{i}} J_{n_{i+d}} \subset F_{i+d}$. Since $J_{n_{i+d}}$ is the $\left(n_{i+2 d}-1\right)$-nearest homterval, we conclude $n_{i+2 d} \leq n_{i+d}+\left(n_{i+d}-n_{i}\right)$. Thus, the sequence $\left\{n_{i+d}-n_{i}\right\}_{i}$ is non-increasing. Hence by $(1.16)$ the critical point $c_{j}$ is periodic $($ where $j \equiv i(\bmod d)$ ). This contradiction completes the proof of Main Theorem for $f \in \mathcal{O}$.

\subsection{Concluding remark}

If assumption $\mathrm{A}$ does not hold then one must modify the argument as follows. Let $\Gamma$ be the set of all homtervals ending at critical points. If $J \in \Gamma$ and $J_{n}$ lies nearer to $c$ than all homtervals $I_{m} \neq J_{n}, m \leq n$, then we call $J_{n}$ the strongly nearest to $c$ homterval. To prove Main Theorem without assumption A we consider such homtervals instead of the nearest homtervals defined above. The similar argument was used in $[3,4]$.

A simpler approach in the smooth case was proposed by the referee. This will be described in Part 2. 
I am grateful to A. M. Blokh for useful discussions of the results of Part 1.

\section{REFERENCES}

[1] A. M. Blokh. Decomposition of dynamical systems on an interval. Russian Math. Surv. 38 N5 (1983), 133-134.

[2] A. M. Blokh. On dynamical systems on one dimensional branched manifolds (in Russian), I, II, 1II. Theory Funct., Functional Anal. \& Appl. (Kharkov) 46 ( 1986), 8-18; 47 (1987), 67-77; 48 (1987), 32-46.

[3] A. M. Blokh \& M. Yu. Lyubich. Attractors of maps of the interval . Func. Anal. \& Appl. 21 N2 (1987), 70-71.

[4] A. M. Blokh and M. Yu. Lyubich. On typical behaviour of trajectories of transformations of the interval (in Russian). Theory Funct., Functional Anal. \& Appl, Kharkov 49 (1988), 5-16.

[5] A. M. Blokh \& M. Yu. Lyubich. Ergodicity of transitive unimodal transformations of the interval. Ukranian Math. J., to appear.

[6] E. M. Coven \& Z. Nitecki. Non-wandering sets of the powers of maps of the interval. Ergot. Th. \& Dynam. Sys. 1 N1 (1981), 10-31.

[7] P. Fatou. Sur les equations fonctionnelles. Bull. Soc. Math. France 48 (1920), 33-94.

[8] M. Feigenlaum. Quantitative universality for a class of non-linear transformations. J. Stat. Phys. 19 (1978), 25-52.

[9] G. M. Golusin. Geometric Theory of Functions of Complex Variable. GITTL: Moscow-Leningrad, 1952.

[10] J. Guckenheimer. Sensitive dependence to initial conditions for one dimensional maps. Comm. Math. Phys. 70 N2 (1979), 133-160.

[11] J. Guckenheimer. Limit sets of S-unimodal maps with zero entropy Comm. Math. Phys. 110 N4 (1987), 655-659.

[12] G. R. Hall. A C $C^{\infty}$-Denjoy counterexample. Ergod. Th. \& Dynam. Sys. 1 N3 (1981), 261-272.

[13] M. V. Jacobson. On smooth mappings of the circle into itself (in Russian). Math. Sbornik. 14 N2 (1971), 163-188.

[14] M. Ju. Lyubich. On typical behaviour of the trajectories of a rational mappings of the sphere. Soviet Marh. Dokl. 27 (1983), 22-25.

[15] W. de Melo and S. van Strien. A structure theorem in one dimensional dynamics. Preprint IMPA, Sér. A-063 (1986).

[16] J. Milnor. On the concept of attractor. Comm. Math. Phys. 99 (1985), 177-195.

[17] M. Misiurewicz. Structure of mappings of an interval with zero entropy. Preprint IHES/M/78/249 (1978).

[18] Z. Nitecki. Differentiable Dynamics. MIT Press: Cambridge, Massachusetts-London, 1971.

[19] A. N. Sharkovskii. Partially ordered system of limit sets (in Russian). Soviet Math. Dokl. 170 (1966), 1276-1278.

[20] D. Singer. Stable orbits and bifurcations of maps of the interval. SIAM J. Appl. Math. 35 (1978), 260-267.

[21] D. Sullivan. Quasi conformal homeomorphisms and dynamics. I. Ann. of Math. 122 N3 (1985), 401-418.

[22] S. van Strien. On the bifurcations creating horseshoes. Springer Lect. Notes Math. 898 (1981), 316-351.

[23] A. Schwartz. A generalization of Poincaré-Bendixon theorem on closed two-dimensional manifolds. Amer. J. Math. 85 (1963), 453-458.

[24] J. C. Yoccoz. Il n'y pas de coutre-exemple de Denjoy analitique. C.R. Acad. Sci. Paris. Ser. 1 N7 298 (1984), 141-144.

[25] L. S. Young. A closing lemma on the interval. Inv. Math. 54 N2 (1979), 179-187.

[26] L. Jonker and D. A. Rand. Bifurcations in one dimension, I: The non-wandering set. Inventions Math. 62 (1981), 347-365. 